\title{
Hybrid extracorporeal membrane oxygenation
}

\author{
Alexandre Brasseur ${ }^{1}$, Sabino Scolletta ${ }^{2}$, Roberto Lorusso ${ }^{3}$, Fabio Silvio Taccone ${ }^{1}$ \\ ${ }^{1}$ Department of Intensive Care, Hôpital Erasme, Université Libre de Bruxelles, ULB, Brussels, Belgium; ${ }^{2}$ Department of Anesthesia and Intensive \\ Care, Università di Siena - Azienda Ospedaliera Universitaria Senese, Siena, Italy; ${ }^{3}$ Department of Cardio-Thoracic Surgery, Heart \& Vascular \\ Centre, Maastricht University Medical Centre, Maastricht, The Netherlands \\ Contributions: (I) Conception and design: FS Taccone, R Lorusso; (II) Administrative support: FS Taccone and A Brasseur; (III) Provision of study \\ materials or patients, A Brasseur, R Lorusso; (IV) Collection and assembly of data: A Brasseur; (V) Data analysis and interpretation: All authors; (VI) \\ Manuscript writing: All authors; (VII) Final approval of manuscript: All authors. \\ Correspondence to: Pr. Fabio Silvio Taccone, MD, PhD. Department of Intensive Care, Erasme Hospital, Université Libre de Brxelles (ULB), Route \\ de Lennik, 808, 1070 Brussels, Belgium. Email: ftaccone@ulb.ac.be.
}

\begin{abstract}
Veno-venous (VV) and veno-arterial (VA) extracorporeal membrane oxygenation (ECMO) therapy is widely used in critically ill patients with refractory cardiogenic shock and cardiac arrest or suffering from severe respiratory failure. Besides traditional ECMO cannulation, changes in patients' conditions or the occurrence of specific complications (i.e., cerebral hypoxia or left ventricular dilation) may require modifications in cannulation strategies or the combination of ECMO with additional invasive or minimally invasive procedures, to improve organ function and ECMO efficiency. In this review, we described all these "hybrid" approaches, such as the addition of a third or fourth ECMO cannula to improve venous drainage and/or optimize systemic hemodynamics/oxygenation, or the implementation of surgical or percutaneous unloading of the left ventricle (LV), to reduce cardiac dilation and pulmonary edema. Although few data are still available about the effectiveness of such interventions, clinicians should be aware of these advances in ECMO management to improve the management of more complex cases.
\end{abstract}

Keywords: Extracorporeal life support (ECLS); membrane oxygenation; hybrid; cannulation; intra-aortic balloon pump (IABP); venting

Submitted Feb 21, 2018. Accepted for publication Mar 06, 2018.

doi: 10.21037/jtd.2018.03.84

View this article at: http://dx.doi.org/10.21037/jtd.2018.03.84

\section{Introduction}

The use of extracorporeal life support (ECLS) or extracorporeal membrane oxygenation (ECMO) therapy for temporary, non-intraoperative support of patients with cardiac and/or pulmonary dysfunction has dramatically increased in the last decade (1). In particular, despite the data supporting the impact of such therapy on patients' outcome are not robust yet, patients with acute hypoxemic respiratory failure, refractory cardiac arrest and/or cardiogenic shock remain the best potential indications for ECMO implementation (2). Nevertheless, prolonged ECMO support, in particular for patients waiting for lung transplant or permanent ventricular assist devices, has required the development of newer technologies and approaches to ensure an adequate patients' management and to reduce the risk of complications. In particular, cannulation strategies have evolved from "basic" venovenous (VV) or veno-arterial (VA, either central or peripheral) configurations to more complex "hybrid" ECMO compositions, with the use of a third or fourth cannula to improve systemic oxygenation or cardiac unloading (3). Moreover, cardiac unloading, in particular for patients with cardiogenic shock, can also be performed using additional devices, such as intra-aortic balloon pump (IABP) counterpulsation or short-term assist devices, which further complicates the ECMO configuration and management for physicians (4). This suggests the need for carefully considering the use of such "hybrid" therapies in very selected patients, in experienced centers and with a 
consistent nomenclature to adequately promote clinical and research practices.

Thus, the aim of this review is to outline "hybrid" ECMO use, including either cannulation strategies different than VA and $\mathrm{VV}$, and to highlight more complex ECMO configurations, using additional support devices to overcome ongoing clinical problems or to improve ECMO efficiency.

\section{"Hybrid" cannulations}

ECMO VA and VV configuration depends on the nature of the underlying disease and the severity of illness. In case of severe cardiogenic shock or inadequate cardiac output from the native heart (i.e., septic cardiomyopathy), VA ECMO should be implemented as main circulatory/respiratory support. In case of severe hypoxemia and/or hypercapnic respiratory failure despite optimized mechanical ventilation and additional therapeutics (i.e., recruitment manoeuvres or prone positioning), VV (either "low-flow" or "high flow") ECMO is the first choice to work as an artificial lung, but without hemodynamic support (5).

Nevertheless, the degree of concomitant heart and lung failure in the same patient may affect the effectiveness of ECMO therapy. As an example, venous drainage may be limited in hypovolemic patients or with small venous size who require high ECMO blood flow, either for circulatory and/or respiratory supply. Also, in patients on VA ECMO with peripheral cannulation, recovery of heart function and persistent severe lung injury would result into the socalled "differential hypoxia". This condition consists in the ejection of poorly oxygenated blood into the ascending aorta from the left ventricle (LV), which competes with retrograde oxygenated blood flow from the ECMO circuit; the potential consequence of differential hypoxia is myocardial and cerebral ischemia (6). Finally, patients on VV ECMO may have a secondary hemodynamic deterioration, particularly right ventricular failure, and require circulatory support. As such, the use of a modified ECMO configuration during support or an "extra" cannula might be considered and implemented to increase draining capability or to address new perfusion needs based on systemic oxygenation or modified ventricular function in this setting.

\section{VA venous (VAV) ECMO}

VAV configuration has been proposed in patients with differential hypoxia or secondary heart failure after VV ECMO initiation (Figure 1). In patients with differential hypoxia (i.e., on VA ECMO), an additional cannula is introduced into the jugular (or subclavian) vein to deliver oxygenated blood to the pulmonary circulation. In patients with secondary heart failure (i.e., on VV ECMO), adequate circulatory support is achieved by adding an extra arterial cannula, either femoral or subclavian/axillary.

In one study, Werner et al. described the use of VAV ECMO in 31 patients, 8 of them were paediatric cases (7). The main reasons for conversion to the VAV mode in adult patients were heart failure (6/13), differential hypoxia (5/13) and worsening of hypoxemia (2/13), with 10 patients having VAV ECMO as initial configuration (Table 1). In one patient, the use of a double lumen jugular cannula was used to minimize the number of cannulated sites. In paediatric patients, 5 were on VA, 2 on VV and 1 had an initial VAV ECMO. Mortality rate was $61 \%$ in adults and $29 \%$ in paediatric patients and the occurrence of neurological complication was $13 \%$ in adults and $29 \%$ in children, respectively. In a second study, Biscotti et al. reported their experience in 21 patients who ultimately received a VAV configuration, 8/21 for secondary heart failure, 2/21 for differential hypoxia and 11/21 having an initial VAV cannulation mode (8). Mortality rate was $57 \%$, with 8 patients dying on ECMO and 4 after ECMO weaning; mortality was also similar in patients with initial VAV configuration when compared to those subsequently converted $(7 / 11,63 \%$ vs. $5 / 10,50 \%)$. Moreover, in one patient a more complex ECMO cannulation including a double venous drainage (VVAV) was implemented to obtain an adequate ECMO blood flow. In another study, Ius et al. treated 10 patients with VAV configuration over a cohort of 406 ECMO supports (9); in this series, 9/10 patients were converted from an initial $\mathrm{VV}$ mode, and mortality was $50 \%$, with 3 patients being bridged to lung transplantation and frequent non-cerebral complications (severe bleeding in 3 and leg ischemia in 2 patients). Also, Stöhr et al. used VAV configurations in 11 patients suffering from acute respiratory distress syndrome (ARDS), and showed a lower mortality rate $(27 \%$ vs. $68 \%)$ when compared to other ARDS patients treated with VV $(\mathrm{n}=11)$ or VA $(\mathrm{n}=8)(10)$.

\section{Other cannulation modes}

More complex veno-veno-venous (VVV), veno-venoarterial (VVA) or veno-veno-arterio-venous (VVAV) configurations are anectodical and no case series have 
A

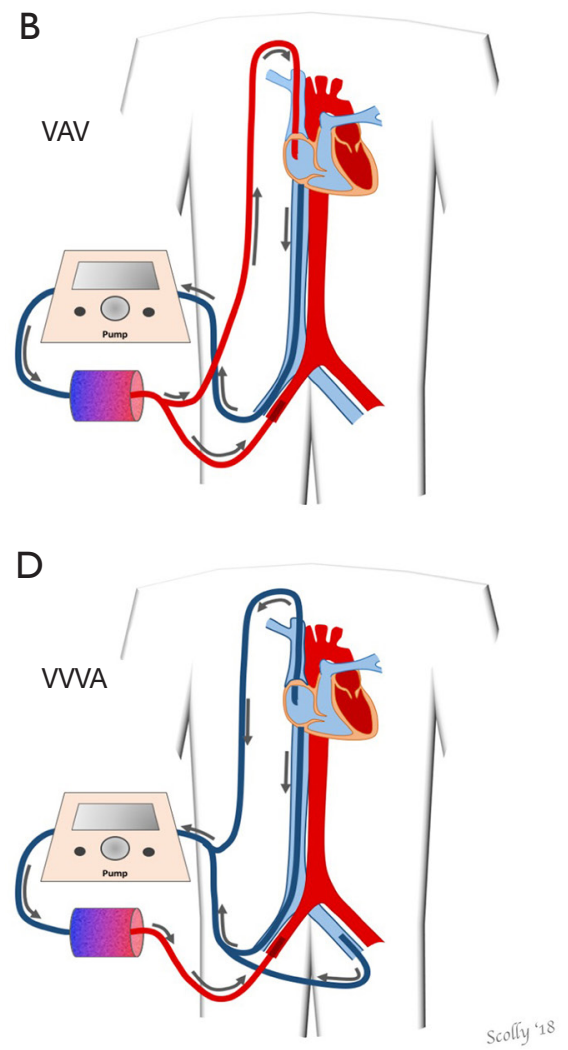

Figure 1 Different type of extracorporeal membrane oxygenation (ECMO) "hybrid" cannulation modes. (A) Veno-venous-arterial (VVA) ECMO with double venous cannulation (combination of venous access is variable) for drainage and femoral artery cannulation for perfusion. (B) Venous-arterial-venous (VAV) ECMO with single venous drainage and right femoral artery and right internal jugular vein for perfusion. (C) Veno-veno-venous-arterial (VVVA) with double-lumen cannula acting only as venous drainage and right femoral artery as perfusion; (C) veno-venous-arterio-venous (VVAV) ECMO with double venous cannulation and right femoral artery and vein as perfusion; (D) VVVA with triple venous drainage and femoral artery as perfusion.

Table 1 Characteristics of studies evaluating "hybrid" ECMO cannulation

\begin{tabular}{|c|c|c|c|c|c|c|c|c|c|}
\hline Study & $\begin{array}{l}\text { Type of } \\
\text { study }\end{array}$ & Patients & Adult & $\begin{array}{c}\text { Duration } \\
\text { (years) }\end{array}$ & VA; VV; VAV* & ECPR (A/P) & $\begin{array}{l}\text { ECMO weaning } \\
(\mathrm{A} / \mathrm{P})\end{array}$ & $\begin{array}{l}\text { Cerebral } \\
\text { complications }\end{array}$ & Mortality \\
\hline Werner (7) & $\mathrm{R}$ & 31 & 23 (74\%) & 14 & $\begin{array}{l}\text { 12/31 (31\%); } \\
8 / 31(26 \%) ; \\
11 / 31(35 \%)\end{array}$ & $\begin{array}{l}9 / 23(39 \%) \\
3 / 8(38 \%)\end{array}$ & $\begin{array}{l}11 / 23(48 \%) \\
6 / 8(75 \%)\end{array}$ & $\begin{array}{l}3 / 23(13 \%) ; \\
2 / 8(25 \%)\end{array}$ & $\begin{array}{l}14 / 23(61 \%) ; \\
3 / 8(38 \%)\end{array}$ \\
\hline Biscotti (8) & $\mathrm{R}$ & 21 & $21(100 \%)$ & 2 & $\begin{array}{l}8 / 21(38 \%) \\
2 / 21(10 \%) ; \\
11 / 21(52 \%)\end{array}$ & 7/21 (33\%) & NR & NR & $12 / 21(57 \%)$ \\
\hline lus (9) & $\mathrm{R}$ & 10 & $10(100 \%)$ & 3 & $\begin{array}{l}9 / 10(90 \%) \\
1 / 10(10 \%)\end{array}$ & 0 & $7 / 10$ (70\%) & 0 & $5 / 10(50 \%)$ \\
\hline Stöhr (10) & $P$ & 11 & $11(100 \%)$ & 3 & $\begin{array}{l}3 / 11(27 \%) \\
5 / 11(45 \%) \\
3 / 11(27 \%)\end{array}$ & 0 & NR & NR & $3 / 11$ (27\%) \\
\hline
\end{tabular}

*, as initial ECMO configuration. A/P, adult/pediatric; NR, not reported; ECMO, extracorporeal membrane oxygenation; VV, Veno-venous; VA, veno-arterial; VAV, VA venous; ECPR, extracorporeal cardiopulmonary resuscitation; R, retrospective; P, prospective. 
been published in the literature yet to describe the main indications, management, complications and outcome. Their use combines the need for a higher venous drainage (double venous cannula) with the possibility to provide both a circulatory and respiratory support (i.e., arterial and/or venous cannulation) at a higher total ECMO blood flow. Double venous drainage could be implemented in case of limited ECMO blood flow, excessive hemolysis or congenital heart defects with right-to-left shunts (11). The use of double lumen venous cannula, inserted in most of cases in the right jugular vein, combined with an arterial reinjection cannula or another venous drainage cannula would also result in a VAV or VVV ECMO.

An additional recent "hybrid" ECMO cannulation is the insertion, either percutaneously or surgically using angiographic or trans-oesophageal echocardiographic guidance, of a long venous cannula into the pulmonary artery (in general via the right jugular vein, the left subclavian vein or the femoral veins) to provide a similar support as VV ECMO (i.e., blood oxygenation and decarboxylation). The main advantage of this so-called "VPa" cannulation or VPa-ECMO is the bypass of the right ventricle, which may be particularly useful in patients with right heart failure and sufficient left ventricular function. Furthermore, the VPa cannula could be easily removed without additional open-chest procedures. This cannulation mode may also act as a percutaneous right ventricular assist device (pRVAD), if no oxygenator is inserted into the system. Also, it can be performed either by using a double cannulation (i.e., a femoral venous drainage and a long reinjection flexible cannula-in general $17 \mathrm{~F}$ of diameter-into the pulmonary artery) or a doublelumen single cannula, with the drainage opening in the right atrium (12). Finally, VA ECMO could be combined with VPa cannulation (i.e., VAVPa ECMO) providing a sort of percutaneous bilateral assist support together with oxygenated and decarboxylated blood into both systemic and pulmonary circulations; this variant has not been validated in clinical studies.

\section{Hybrid configurations}

Hybrid ECMO configurations have been reported in particular for VA ECMO, either in case of severe cardiogenic shock or after extracorporeal cardiopulmonary resuscitation (ECPR). One of the most important issues during the use of VA ECMO is the effect of retrograde aortic flow due to peripheral femoral cannulation, which increases LV afterload and potentially impacts on LV function and recovery (13). The consequences of increased $\mathrm{LV}$ afterload are ventricular dilation, increased left atrial pressure and pulmonary edema. These phenomena are even more striking in case of previous heart failure with concomitant mitral valve regurgitation or previous aortic regurgitation, which significantly raise the risk of LV and alveolar overload (14). Moreover, in case of severe LV dysfunction, the retrograde VA ECMO blood flow may promote the closing of the aortic valve during systole, which would result in intra-ventricular blood stasis and thrombi formation (15). Finally, LV overload increases myocardial oxygen consumption and would increase the risk of ischemic lesions, particularly in the subendocardial regions, and ventricular stunning (16). Taken together, all these findings suggest that unloading the LV during VA ECMO may provide $L V$ functional rest, reduce the risk of ventricular wall stress and pulmonary edema, and favour heart recovery. Nevertheless, such adverse effects are not observed in all patients undergoing VA ECMO and unloading of the LV may require additional invasive procedures, which may also increase the risk of complications. Thus, the balance between risk and benefits of $L V$ unloading as well as the prompt identification of the best candidates should always be considered when more complex ECMO configurations are proposed to patients on VA ECMO.

\section{Surgical "bybrid" configurations}

Left ventricular venting can be achieved through a surgical insertion of a catheter, either during or immediately after heart surgery. In case of peripheral VA ECMO support in patients suffering from post-cardiotomy heart failure, the detection of $\mathrm{LV}$ overload can require the shift to a central VA ECMO configuration and the insertion of a $L V$ venting catheter through the cannulation of the left atrium or ventricle via the right superior pulmonary vein, which is then connected by a Y-tubing to the venous drainage line of the ECMO circuit. Interestingly, this procedure of surgical $\mathrm{LV}$ venting has been associated with a high rate of cardiac recovery and subsequent successful ECMO weaning in both paediatric and adult patients $(17,18)$. In paediatric VA ECMO, an alternative to this approach is to place the $\mathrm{LV}$ venting cannula into the pulmonary artery trunk (because of the small size of pulmonary veins observed in these patients), the direct left atrium cannulation placed through a trans-thoracic approach or through the surgical exposure of the groove between the pulmonary veins and right atrium 
(i.e., the Waterston's groove) (19,20).

In case of peripheral VA ECMO configuration, LV unloading may be performed using minimally invasive surgical techniques. One approach is to expose the left apex through a surgical incision and to place into the $\mathrm{LV}$ a $16-20 \mathrm{~F}$ cannula, which is subsequently tunnelled via a subxiphoidal incision (i.e., the "subxiphoidal" approach); this technique is used in particular for patients who underwent heart surgery with sternotomy (21). The second approach consists in a left anterolateral thoracotomy, with the venting placed into the LV apex using fluoroscopy or echocardiography (22). In one study, Centofanti et al. described the combination of VA ECMO and trans-apical LV venting through a left minithoracotomy and connected to the venous drainage line of ECMO (23); in all patients, hemodynamics improved, recovery of heart function was observed in $46 \%$ of cases and 30 -day mortality was $38 \%$. These procedures require a high surgical expertise and potentially increase the risk of myocardial wall damage or coronary artery injuries, as the risk of bleeding from the chest incision, as the patients receive systemic anticoagulation.

\section{Percutaneous "bybrid" configurations}

LV unloading can also be achieved by several percutaneous approaches. First, a pulmonary drainage, consisting in a venous cannula placed into the main pulmonary artery and connected to the drainage ECMO cannula, has been described in two cases, one concerning a refractory cardiogenic shock after pulmonary sepsis in an adult patient and the other with restrictive cardiomyopathy into a young woman (24,25). Second, a trans-aortic catheter venting, using a 5-7 F drainage cannula placed across the aortic valve directly into the LV cavity using a trans-femoral approach, can be achieved under echocardiography guidance and connected to the venous ECMO circuit. In one case, a 17 F paediatric cannula was placed into the $\mathrm{LV}$ cavity using the same technique via the subclavian artery $(26,27)$. Third, a left-to-right shunt due to atrial communication may positively influence the LV unloading; this communication can be obtained through percutaneous techniques that artificially create the shunting. In one study, Aiyagari et al. incorporated left atrium drainage into the ECMO venous circuit in 7 paediatric patients via a trans-septal puncture and the placement of a left atrial 8-15 F cannula; no major complication was reported and successful ECMO weaning was obtained in 4 patients (28). Left-to-right atrial communication can also be obtained using blade and balloon septostomy, which, in one study, was feasible in all 9 cases and led to significant $\mathrm{LV}$ and pulmonary unloading with a survival rate of $70 \%$ (29). Atrial communication patency can be maintained using specific stentings, which may help to titrate the atrial shunt and preserve an adequate flow over time, although this approach may produce some complications because of anatomical injury and the need for surgical correction at the moment of venting removal (30).

\section{"Hybrid" configuration with additional devices}

The use of IABP can decrease LV afterload, increase diastolic blood pressure and coronary blood flow (Figure 2). In one study, 144 of 219 patients requiring VA ECMO after cardiac surgery were concomitantly treated with IABP (31); the use of IABP was associated with a significant increase in survival rate. In another study, Gass et al. described a cohort of 135 patients who underwent peripheral VA ECMO and concomitant IABP implantation. Main complications included local bleeding (14\%) and stroke (11\%); overall inhospital survival was $58 \%$, with some patients being bridged to heart transplantation (3\%) or left ventricular assist device (LVAD) (15\%) and $41 \%$ of patients showing myocardial recovery. IABP use was an independent predictor of reduced in-hospital mortality and complications (32). In another study, including patients requiring VA ECMO after heart transplantation, the use of IABP was also associated with higher weaning and survival rates (33). Moreover, improved hemodynamics, including higher mean arterial pressure, lower central venous and wedge pressures, reduction in lactate and increase in venous oxygen saturation levels, have been reported in other studies when IABP was added to VA ECMO therapy $(34,35)$. Nevertheless, other studies reported less positive results. Ro et al. did not observe an increased survival rate, despite a greater number of patients being weaned from extracorporeal support, with the use of IABP during VA ECMO (36). Also, no improvement in survival with the combination of IABP and VA ECMO was observed in two other studies $(37,38)$. Moreover, the addition of IABP to VA ECMO resulted in different effects on cerebral hemodynamics; IABP decreased cerebral blood flow (CBF) during cardiac stunning while it increased $\mathrm{CBF}$ in case of heart recovery (i.e., pulsatile pressure $>10$ $\mathrm{mmHg}$ ) (39). Finally, in a recent meta-analysis, IABP was not associated with improved outcome if associated with VA ECMO in different subgroups of patients with severe heart failure (40).

An alternative to IABP is the use of temporary 
A ECMO VA
IABP

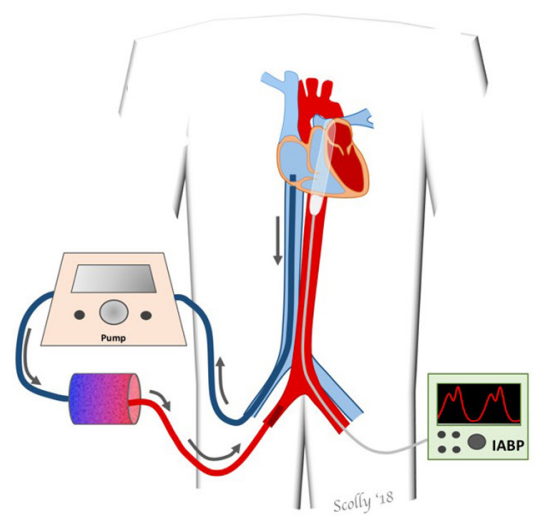

B

$B$

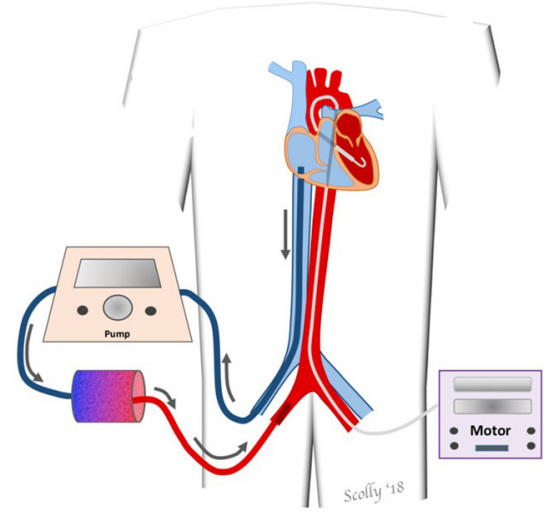

C

ECMO VA

Tandem heart

Figure 2 Percutaneous mechanical circulatory support devices, such as intra-aortic balloon counterpulsation (IABP) (A), Impella (B) or Tandem-Heart (C), in combination with veno-arterial ECMO. ECMO, extracorporeal membrane oxygenation; VA, veno-arterial.

extracorporeal LVADs, which can be implanted using a minimally invasive percutaneous femoral procedure. The Impella (Abiomed Inc., USA) is a catheter, which is associated with a trans-aortic axial flow pump that can drain blood from the LV through an inlet area and reinject it into the ascending aorta thus producing a blood flow of 2.5 to $5.0 \mathrm{~L} / \mathrm{min}$. In adults, Pappalardo et al. reported about 34 of 157 patients on VA ECMO who received concomitant treatment with the Impella; using a propensity score, 21 patients on Impella and VA ECMO showed a significant lower mortality rate $(47 \%$ vs. $80 \%)$ than matched patients on VA ECMO alone (41). Cheng et al. also reported a significant reduction in end-diastolic $\mathrm{LV}$ diameter with the implantation of Impella to VA ECMO (42). Also, Eliet et $a l$. showed that Impella could improve pulmonary flow and ensure effective LV discharge during VA ECMO therapy (43). The Impella device has been also associated with VPa cannulation to provide effective biventricular support in case of severe cardiogenic shock (44). Finally, Impella implantation during VA ECMO is as effective as surgical LV venting to promote ventricular and pulmonary unloading, with similar outcome and complications (45).

The Tandem-Heart (Cardiac Assist, Inc., Pittsburgh, PA, USA) consists of two devices: the first one implies a percutaneous cannula via the femoral vein that is placed via an atrial septostomy to drain blood from the left atrium and the second reinjecting blood into the aorta via a femoral artery access. A small case series has described the combination of Tandem-Heart and VA ECMO in 5 patients as a valuable option to unload the $\mathrm{LV}$, although survival rate was very poor (46). In another case, VA ECMO was combined with biventricular unloading via bi-atrial cannulation using a Tandem-Heart device in the presence of LV thrombus during refractory cardiac arrest (47). A second cannula (Protek-Duo, CardiacAssist, Pittsburgh, PA, USA) includes a double-lumen cannula positioned into the pulmonary artery via the internal jugular vein and drainage from the right atrium and reinfusion into the pulmonary artery. This configuration should combine the advantages of single cannulation with right ventricular bypass.

\section{Limitations}

Cannulation strategies in ECMO patients may not be fixed during extracorporeal support and physicians should promptly recognize changes in patient's physiology or clinical conditions, which may require to upgrade to more complex cannulation or configurations. The conversion from the initial to a different ECMO mode should be accurately evaluated and considered with caution due to the ongoing anticoagulation for the ECMO run, with higher risk of bleeding, particularly in case of arterial cannulation or chest (re)opening. Furthermore, the choice of a third or fourth cannula or for an additional percutaneous or surgical procedure may represent another port for infection or thrombosis. Also, to adequately distribute the ECMO blood flow between the different reinjection cannulas (i.e., arterial and venous) in the VAV mode, a Hoffman clamp 
is generally used, although this requires an accurate flow monitoring and could increase the risk of hemolysis.

\section{Conclusions}

Together with the traditional VA and VV ECMO modes, there are a number of different and more complex ECMO cannulation strategies and configurations increasingly considered during the ECMO course. Triple cannulation can be useful to improve venous drainage or combine both respiratory and circulatory support in case of concomitant lung and heart dysfunction. VPa cannulation is a novel modification of VV ECMO to provide respiratory support in case of right ventricular failure. For LV unloading, both surgical and percutaneous procedures are available. The combination of VA ECMO with IABP, Impella or TandemHeart may also be valuable options to promote effective $\mathrm{LV}$ or right ventricular unloading. The development of new cannulas and the better knowledge of "hybrid" cannulation and configurations will broaden the clinical options to manage complex cases of severe cardiac and/or pulmonary failure.

\section{Acknowledgements}

None.

\section{Footnote}

Conflicts of Interest: The authors have no conflicts of interest to declare.

\section{References}

1. Gerke AK, Tang F, Cavanaugh JE, et al. Increased trend in extracorporeal membrane oxygenation use by adults in the United States since 2007. BMC Res Notes 2015;8:686.

2. Tramm R, Ilic D, Davies AR, et al. Extracorporeal membrane oxygenation for critically ill adults. Cochrane Database Syst Rev 2015;1:CD010381.

3. Sorokin V, MacLaren G, Vidanapathirana PC, et al. Choosing the appropriate configuration and cannulation strategies for extracorporeal membrane oxygenation: the potential dynamic process of organ support and importance of hybrid modes. Eur J Heart Fail 2017;19 Suppl 2:75-83.

4. Meani P, Gelsomino S, Natour E, et al. Modalities and Effects of Left Ventricle Unloading on Extracorporeal Life support: a Review of the Current Literature. Eur J Heart Fail 2017;19 Suppl 2:84-91.

5. Combes A, Brodie D, Chen YS, et al. The ICM research agenda on extracorporeal life support. Intensive Care Med 2017;43:1306-18.

6. Loftsgard TO, Newcome MD, Hanneman MR, et al. Management of Neurogenic Pulmonary Edema and Differential Hypoxemia in an Adult Supported on Venoarterial Extracorporeal Membrane Oxygenation. J Cardiothorac Vasc Anesth 2017;31:2170-4.

7. Werner NL, Coughlin M, Cooley E, et al. The University of Michigan experience with venoveno-arterial hybrid mode of extracorporeal membrane oxygenation. ASAIO J 2016;62:578-83.

8. Biscotti M, Lee A, Basner RC, et al. Hybrid configurations via percutaneous access for extracorporeal membrane oxygenation: a single centre experience. ASAIO J 2014;60:635-42.

9. Ius F, Sommer W, Tudorache I, et al. Veno-veno-arterial extracorporeal membrane oxygenation for respiratory failure with severe haemodynamic impairment: technique and early outcomes. Interact Cardiovasc Thorac Surg 2015;20:761-7.

10. Stöhr F, Emmert MY, Lachat ML, et al. Extracorporeal membrane oxygenation for acute respiratory distress syndrome: is the configuration mode an important predictor for the outcome? Interact Cardiovasc Thorac Surg 2011;12:676-80.

11. Ford EG, Atkinson JB. Augmented venous access in the problematic ECMO patient: a case report. J Pediatr Surg 1992;27:527-8.

12. Kazui T, Tran PL, Echeverria A, et al. Minimally invasive approach for percutaneous CentriMag right ventricular assist device support using a single PROTEKDuo Cannula. J Cardiothorac Surg 2016;11:123.

13. Ostadal P, Mlcek M, Kruger A, et al. Increasing venoarterial extracorporeal membrane oxygenation flow negatively affects left ventricular performance in a porcine model of cardiogenic shock. J Transl Med 2015;13:266.

14. Soleimani B, Pae WE. Management of left ventricular distension during peripheral extracorporeal membrane oxygenation for cardiogenic shock. Perfusion 2012;27:326-31.

15. Aissaoui N, Guerot E, Combes A, et al. Two-dimensional strain rate and Doppler tissue myocardial velocities: analysis by echocardiography of hemodynamic and functional changes of the failed left ventricle during different degrees of extracorporeal life support. J Am Soc Echocardiogr 2012;25:632-40. 
16. Koeckert MS, Jorde UP, Naka Y, et al. Impella LP 2.5 for left ventricular unloading during venoarterial extracorporeal membrane oxygenation support. J Card Surg 2011;26:666-8.

17. Sandrio S, Springer W, Karck M, et al. Extracorporeal life support with an integrated left ventricular vent in children with a low cardiac output. Cardiol Young 2014;24:654-60.

18. Weymann A, Schmack B, Sabashnikov A, et al. Central extracorporeal life support with left ventricular decompression for the treatment of refractory cardiogenic shock and lung failure. J Cardiothorac Surg 2014;9:60

19. Kotani Y, Chetan D, RodriguesW, et al. Left atrial decompression during venoarterial extracorporeal membrane oxygenation for left ventricular failure in children: current strategy and clinical outcomes. Artif Organs 2013;37:29-36.

20. Hacking DF, Best D, d'Udekem Y, et al. Elective decompression of the left ventricle in pediatric patients may reduce the duration of venoarterial extracorporeal membrane oxygenation. Artif Organs 2015;39:319-26.

21. Guirgis M, Kumar K, Menkis AH, et al. Minimally invasive left-heart decompression during venoarterial extracorporeal membrane oxygenation: an alternative to a percutaneous approach. Interact Cardiovasc Thorac Surg 2010;10:672-4.

22. Cheung A, Lichtenstein KM. Illustrated techniques for transapical aortic valve implantation. Ann Cardiothorac Surg 2012;1:231-9.

23. Centofanti P, Attisani M, La Torre M, et al. Left Ventricular Unloading during Peripheral Extracorporeal Membrane Oxygenator Support: A Bridge To Life In Profound Cardiogenic Shock. J Extra Corpor Technol 2017;49:201-5.

24. Avalli L, Maggioni E, Sangalli F, et al. Percutaneous leftheart decompression during extracorporeal membrane oxygenation: an alternative to surgical and transeptal venting in adult patients. ASAIO J 2011;57:38-40.

25. Fouilloux V, Lebrun L, Mace L, et al. Extracorporeal membranous oxygenation and left atrial decompression: a fast and minimally invasive approach. Ann Thorac Surg 2011;91:1996-7.

26. Hong TH, Byun JH, Lee HM, et al. Initial Experience of Transaortic Catheter Venting in Patients with Venoarterial Extracorporeal Membrane Oxygenation for Cardiogenic Shock. ASAIO J 2016;62:117-22.

27. Fumagalli R, Bombino M, Borelli M, et al. Percutaneous bridge to heart transplantation by venoarterial ECMO and transaortic left ventricular venting. Int J Artif Organs
2004;27:410-3.

28. Aiyagari RM, Rocchini AP, Remenapp RT, et al. Decompression of the left atrium during extracorporeal membrane oxygenation using a transseptal cannula incorporated into the circuit. Crit Care Med 2006;34:2603-6.

29. Seib PM, Faulkner SC, Erickson CC, et al. Blade and balloon atrial septostomy for left heart decompression in patients with severe ventricular dysfunction on extracorporeal membrane oxygenation. Catheter Cardiovasc Interv 1999;46:179-86.

30. Haynes S, Kerber RE, Johnson FL, et al. Left heart decompression by atrial stenting during extracorporeal membrane oxygenation. Int J Artif Organs 2009;32:240-2.

31. Doll N, Kiaii B, Borger M, et al. Five-year results of 219 consecutive patients treated with extracorporeal membrane oxygenation for refractory postoperative cardiogenic shock. Ann Thorac Surg 2004;77:151-7.

32. Gass A, Palaniswamy C, Aronow WS, et al. Peripheral venoarterial extracorporeal membrane oxygenation in combination with intra-aortic balloon counterpulsation in patients with cardiovascular compromise. Cardiology 2014;129:137-43.

33. Santise G, Panarello G, Ruperto C, et al. Extracorporeal membrane oxygenation for graft failure after heart transplantation: a multidisciplinary approach to maximize weaning rate. Int J Artif Organs 2014;37:706-14.

34. Bréchot $\mathrm{N}$, Demondion $\mathrm{P}$, Santi F, et al. Intra-aortic balloon pump protects against hydrostatic pulmonary oedema during peripheral venoarterial-extracorporeal membrane oxygenation. Eur Heart J Acute Cardiovasc Care 2018;7:62-9.

35. Ma P, Zhang Z, Song T, et al. Combining ECMO with IABP for the treatment of critically Ill adult heart failure patients. Heart Lung Circ 2014;23:363-8.

36. Ro SK, Kim JB, Jung SH, et al. Extracorporeal life support for cardiogenic shock: influence of concomitant intraaortic balloon counterpulsation. Eur J Cardiothorac Surg 2014;46:186-92.

37. Park TK, Yang JH, Choi SH, et al. Clinical impact of intra-aortic balloon pump during extracorporeal life support in patients with acute myocardial infarction complicated by cardiogenic shock. BMC Anesthesiol 2014;14:27.

38. Lin LY, Liao CW, Wang CH, et al. Effects of Additional Intra-aortic Balloon Counter-Pulsation Therapy to Cardiogenic Shock Patients Supported by Extra-corporeal Membranous Oxygenation. Sci Rep 2016;6:23838. 
39. Yang F, Jia ZS, Xing JL, et al. Effects of intra-aortic balloon pump on cerebral blood flow during peripheral venoarterial extracorporeal membrane oxygenation support. J Transl Med 2014;12:106.

40. Cheng R, Hachamovitch R, Makkar R, et al. Lack of Survival Benefit Found With Use of Intraaortic Balloon Pump in Extracorporeal Membrane Oxygenation: A Pooled Experience of 1517 Patients. J Invasive Cardiol 2015;27:453-8.

41. Pappalardo F, Schulte C, Pieri M, et al. Concomitant implantation of Impella(R) on top of veno-arterial extracorporeal membrane oxygenation may improve survival of patients with cardiogenic shock. Eur J Heart Fail 2017;19:404-12.

42. Cheng A, Swartz MF, Massey HT. Impella to unload the left ventricle during peripheral extracorporeal membrane oxygenation. ASAIO J 2013;59:533-6.

43. Eliet J, Gaudard P, Zeroual N, et al. Effect of Impella During Veno-Arterial Extracorporeal Membrane Oxygenation on Pulmonary Artery Flow as Assessed by

Cite this article as: Brasseur A, Scolletta S, Lorusso R, Taccone FS. Hybrid extracorporeal membrane oxygenation. J Thorac Dis 2018;10(Suppl 5):S707-S715. doi: 10.21037/ jtd.2018.03.84
End-Tidal Carbon Dioxide. ASAIO J 2017. [Epub ahead of print].

44. Sayed S, Schimmer C, Shade I, et al. Combined pulmonary and left ventricular support with veno-pulmonary ECMO and impella 5.0 for cardiogenic shock after coronary surgery. J Cardiothorac Surg 2017;12:38.

45. Tepper S, Masood MF, Baltazar Garcia M, et al. Left Ventricular Unloading by Impella Device Versus Surgical Vent During Extracorporeal Life Support. Ann Thorac Surg 2017;104:861-7.

46. Li YW, Rosenblum WD, Gass AL, et al. Combination use of a TandemHeart with an extracorporeal oxygenator in the treatment of five patients with refractory cardiogenic shock after acute myocardial infarction. Am J Ther 2013;20:213-8

47. Jumean M, Pham DT, Kapur NK. Percutaneous biatrial extracorporeal membrane oxygenation for acute circulatory support in advanced heart failure. Catheter Cardiovasc Interv 2015;85:1097-9. 